\title{
Brain connectivity in continuous error tasks
}

\author{
Jason Omedes, Iñaki Iturrate, Luis Montesano
}

\begin{abstract}
Error-related potentials (ErrP) have been recently incorporated in brain-machine interfaces (BMIs) due to its ability to adapt and correct both the output of the BMI or the behavior of the machine. Most of these applications rely on synchronous tasks with different user's evaluations associated to correct and wrong events. Asynchronous detection during the continuous evaluation of the task, however, has to cope with background noise and an increased number of misdetections common in event-related potential detection. This paper studies a different characteristic that may carry additional information to be exploited by asynchronous ErrP detectors: brain connectivity coherence patterns appearing while the user monitors the continuous operation of a device. The results obtained with five subject revealed the presence of an error potential in an asynchronous reaching task an showed an increase in the coherency within the theta band.
\end{abstract}

\section{INTRODUCTION}

EEG-based error-related potentials [1]-[3] have received increasing attention in the last years in the field of brainmachine interfaces (BMIs) [4]. These potentials are generated as a result of a mismatch in the user's expected outcome of a task event and the actual one [2]. Since the first work suggesting that they could be detected in single trials [5], they have been successfully used within several BMI applications, such as for the correction of BMI outputs [6], classifier adaptation [7] or for learning sequential tasks [8], [9].

All the previous applications rely on the detection of these potentials using the raw temporal signal (i.e. the amplitude and latency of the ErrP at several channels), which in principle would encode different values for correct and wrong events evaluated by the user. Furthermore, they rely on discrete tasks, where the signals were detected in a synchronous fashion. A recent work has shown that it is possible to asynchronously detect errors during tasks where a device performs continuous trajectories using ECoG [10]. In this asynchronous setting, there are no explicit correct events since the device is continuously moving. Instead, the error has to be distinguished from the background noise. However, as EEG suffers from lower signal-to-noise ratios a non-negligible number of false detections is expected, as indeed happens when classifying other EEG potentials [11].

Consequently, there is a growing research interest in exploring alternative neural correlates of error-related potentials that provide extra information to boost or strengthen the decoders. For instance, error potentials are known to have an associated event-related synchronization in theta band [12] that has been exploited to improve detection rates [13].

Similarly, connectivity features such as coherence have recently proven to be an alternative to temporal and powerspectra features power ones, by offering an additional source

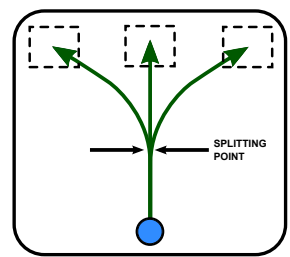

(a)

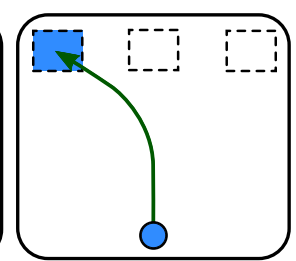

(b)

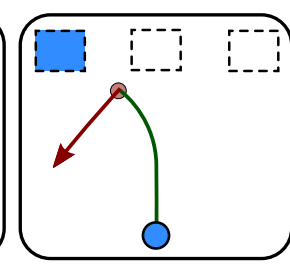

(c)
Fig. 1. Experimental protocol with the device colored in blue and the three possible trajectories (green lines). (b) Example of correct trajectory. (c) Example of an erroneous trajectory.

of information for the classification of ErrPs [14]. These features aim at exploiting the fact that error processing involves the communication between different brain neural sources rather than being generated within a single region [15].

In this paper, we study coherence patterns to characterize the brain connectivity in an asynchronous setup where the user evaluates the performance of a device during continuous trajectories. The results obtained for 5 subjects reveals the presence of an error potential in an asynchronous task, and shows an increase in the coherency within the theta band. Furthermore, phase slope indexes suggest that the signal propagates from central to parietal brain regions.

\section{METHODS}

\section{A. Data Recording}

Electroencephalographic (EEG) and electrooculographic (EOG) activity was recorded using a gTec system consisting of 32 electrodes distributed according to an extended 10/20 international system (FP1, FP2, F7, F8, F3, F4, T7, T8, C3, C4, P7, P8, P3, P4, O1, O2, AF3, AF4, FC5, FC6, FC1, FC2, CP5, CP6, CP1, CP2, Fz, FCz, Cz, CPz, Pz and $\mathrm{Oz}$ ), with the ground on $\mathrm{FPz}$ and the reference on the left earlobe; for the EOG, 6 monopolar electrodes were recorded (placed above and below each eye, and from the outer canthi of the left and right eyes), with the ground on $\mathrm{FPz}$ and the reference on the left mastoid. EEG and EOG signals were digitized with a sampling frequency of $256 \mathrm{~Hz}$, powerline notch filtered at $50 \mathrm{~Hz}$ and high-pass filtered at 0.5 Hz. Additionally, the horizontal, vertical, and radial EOG were computed to remove the EOG from the EEG using a regression algorithm [16]. The data acquisition and on-line processing was developed under a self-made BCI platform.

\section{B. Experimental Setup}

The visual protocol consisted in a blue ball (device) that performed continuous trajectories towards one of three 
possible fixed targets spotted at the top of the screen at left, center and right sides. The possible targets were represented as squares of dashed lines, and the current target was filled in blue (see Figure 1). The device always started at the bottomcenter of the screen. Once a trajectory began, the ball moved continuously towards one of the targets executing a correct or wrong movement, with $70 \%$ and $30 \%$ of probability respectively. Correct trajectories for each target are shown in Fig. 1a. Erroneous trajectories start as correct ones but undergo an instantaneous change of direction, (Figure 1c) at a random point in time after the splitting point.

Five healthy right-handed male subjects (mean age 27 years) voluntarily participated in the study recorded in a laboratory of the University of Zaragoza. Participants were seated on a comfortable chair approximately one meter away from a computer screen where the visual protocol was displayed. Users freely chose one of the three possible targets to reach via three buttons and later observe the trajectories performed by the device continuously evaluating them as correct or erroneous among the whole path to its destination. The users rested as much as needed between targets. Each session was organized in 8 repetitions of 30 movements each, obtaining around 160 correct and 80 erroneous trajectories. Total time per repetition was approximately 4 minutes, plus a break of few minutes between them making the whole duration of the experiments around 45 min per subject.

\section{Connectivity measures}

In order to estimate connectivity measures between the different areas of the brain we computed the coherence $(\mathrm{COH})$ based on multivariate autoregressive (MVAR) models. Let $X(t)=\left[x_{1}(t), x_{2}(t), \ldots, x_{k}(t)\right]^{T}$ denote the EEG recording of $k$ channels at time $t$. The MVAR process can be described as:

$$
X(t)=\sum_{i=1}^{p} A(i) X(t-i)+E(t)
$$

where $E(t)=\left[e_{1}(t), e_{2}(t), \ldots, e_{k}(t)\right]^{T}$ is a vector of multivariate zero-mean uncorrelated white noise process, $A(1), A(2), \ldots A(p)$ are the $k \times k$ matrices of model coefficients and $p$ is the order of the model, which in this study was chosen by the Akaike Information Criterion (AIC) [17] resulting in a $p=11$ order model. Once a MVAR model is estimated, the process of Eq (1) is transformed to the frequency domain:

$$
\begin{gathered}
A(f) X(f)=E(f) \\
X(f)=A^{-1}(f) E(f)=H(f) E(f)
\end{gathered}
$$

where

$$
A(f)=\sum_{k=0}^{p} A(k) e^{-j 2 \pi f \Delta t k}
$$

Here $H(f)$ is the transfer matrix of the model, whose element $H_{i j}(f)$ represents the connection from channel $j$ to channel $i$ at a frequency $f$. Using this expression we can derive different connectivity measures. For this study, the cross-spectral density (S) is obtained by:

$$
S(f)=H(f) \Sigma H(f)^{H}
$$

From here, the coherency (CHY) is the cross spectral density function normalized by individual auto spectral density functions, and can be calculated as:

$$
C H Y_{i j}(f)=\frac{S_{i j}(f)}{\sqrt{S_{i i}(f) S_{j j}(f)}}
$$

where the coherence $(\mathrm{COH})$ is its absolute value

$$
\mathrm{COH}_{i j}(f)=\frac{\left|S_{i j}(f)\right|}{\sqrt{S_{i i}(f) S_{j j}(f)}}
$$

and from the imaginary part of the coherency, we can compute the phase slope index (PSI) as a measure of the information flow [18]:

$$
P S I_{i j}(f)=\Im\left(\sum_{f \in F} C H Y_{i j}^{*}(f) C H Y_{i j}(f+\delta f)\right)
$$

\section{Analysis of continuous error evaluation}

Coherence patterns were computed using a time window of $800 \mathrm{~ms}$ starting at the abrupt change of direction for erroneous trajectories and at the splitting point for correct ones (Figure 1). The MVAR model and corresponding coherence measures were computed for each condition between all pairs of channels (i.e. 991 coherence values). In order to minimize volume conduction effects, coherence was analyzed in terms of statistical differences between error and correct trajectories and displayed at three frequency ranges (theta (3-7 $\mathrm{Hz}$ ), alpha (8-12 Hz) and low beta (12-15 Hz)). In order to average coherence values between subjects the $\mathrm{z}$-Fisher transformation was used. A Wilcoxon rank sum test was used to find the most significant connections between channels at each frequency band and results were plotted in connectivity maps for each channel.

The time evolution of coherence [19] in the theta band (most discriminant band between error and correct events) was analyzed. The EEG data was divided in epochs of 500 $\mathrm{ms}$, sliding in steps of $50 \mathrm{~ms}$ resulting in a $90 \%$ overlap. Signal was standardized for every sliding window (subtracting temporal mean and dividing by the standard deviation) and the difference between error and correct coherence was computed for the most significantly different pairs of channels. For comparison purposes, the grand averaged signals were computed for the error and correct conditions at channel $\mathrm{FCz}$ [8]. Event-related synchronizations (ERS) were calculated in a time window of -1500 to $1500 \mathrm{~ms}$ using Morlet wavelets, with a wavelet-width of 12 cycles. The signal corresponding to a window of time between -1500 to $-500 \mathrm{~ms}$ before the occurrence of the event was used as baseline activity. A statistical significance test based on bootstrapping was run over the ERSs following the method described in [20]. 

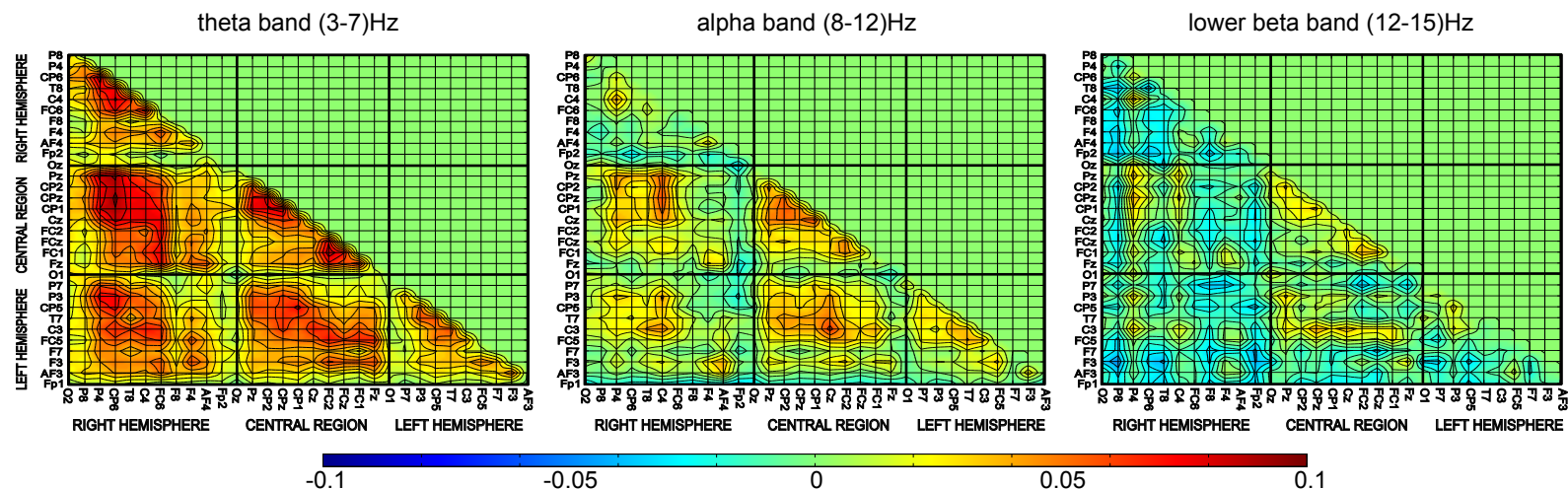

Fig. 2. Fisher-Z transformed coherence patterns of the difference between error and correct conditions for the three different frequency bands. Channels are ordered from occipital to frontal and from right to left brain hemispheres.

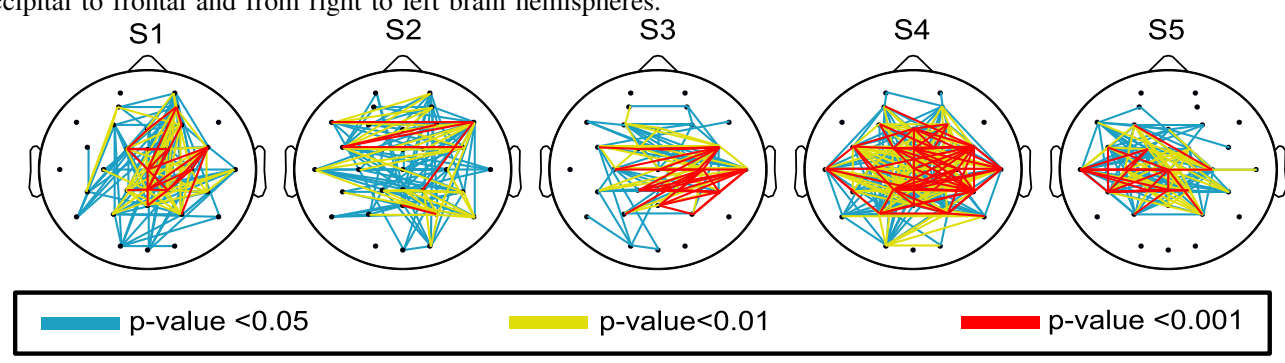

Fig. 3. Results of coherence connectivity maps for the comparisons between the error and correct conditions for each subject at the theta frequency band. Connections depicted in red indicate higher values. Figure is best view in color.

Finally, the phase slope index values between channels were calculated for all electrode pairs for the theta band with frequency resolution $1 \mathrm{~Hz}$ and a time window of $500 \mathrm{~ms}$. The PSI was computed at two different instants of time, one at $-500 \mathrm{~ms}$, when the error has not been presented, and other at $600 \mathrm{~ms}$ corresponding with the interval of time that contains the principal oscillation of the ErrP.

\section{RESULTS}

Figure 2 displays the averaged coherence patterns during the evaluation of the erroneous stimuli in the $800 \mathrm{~ms}$ window. Here, it is evident an increase of coherence distributed into the fronto central and parietal channels at the theta frequency band, as suggested in previous works [12], [14]. Additionally, the averaged coherence also revealed significant connectivity patterns between hemispheres, and to a lower degree, between frontal and parietal areas, despite the large intersubject variability (see figure 3 for the most significant coherences according to the Wilcoxon rank sum analysis $(p<0.05)$ for each subject at the theta frequency band).

Figure 4 shows the temporal evolution of the grand average for the error and correct conditions, the event-related synchronization and the difference (error minus correct) coherence for the most significant electrode pairs at the theta band. As can be seen, an error potential appeared after the sudden change of direction $(0 \mathrm{~ms})$ following the standard characterization described in previous studies [8]. Similarly, the time-frequency ERS shows a power increment in the theta band for the time window between 0 and $1000 \mathrm{~ms}$ [12]. Focusing in the coherency, the five most representative pairs among subjects $(\mathrm{CPz}-\mathrm{CP} 1, \mathrm{Cz}-\mathrm{C} 4, \mathrm{Cz}-$ CP6,Pz-P4 and FC1-CP6) plus one randomly chosen of the non-representative electrode combinations(C4-FP1) are shown. Clearly, the coherence between electrode pairs of fronto-central and parietal brain regions increases for the window of time -200 to $500 \mathrm{~ms}$ when users are evaluating an error, whereas random coherence values remain very low during the entire trajectory.

Finally, Figure 5 depicts the phase slope index values at -500 and $600 \mathrm{~ms}$. As expected, the phase of the different combinations of channels in a given instant of time before the occurrence of the error does not follow a distinguished pattern. On the other hand, looking at the representation obtained from the temporal window that cover the stimuli, there exist a pattern that situate fronto-central channels as the drivers of the error potential, and indicates how the signal propagates towards the parietal areas of the brain.

\section{CONCLUSiOnS AND FUtURE WORK}

This paper has shown the existence of an error potential during continuous and asynchronous tasks, and describes the associated brain connectivity based on coherency measures during error evaluation. We studied coherence changes between 32 electrode combinations, detecting significant increment of coherence between fronto-central and parietal sites at theta frequency band. Furthermore, the phase slope index analysis suggest that the flow of the signal travels from frontal areas (Fz) towards fronto-central and parietal ones. The maximum of coherence difference between correct and erroneous responses reaches its maximum around 600 $\mathrm{ms}$ after the error, which coincides with the larger negative and positive peaks in amplitude of error responses. Future experiments will study how to use coherence measures for feature extraction in order to detect ErrPs in on-line 

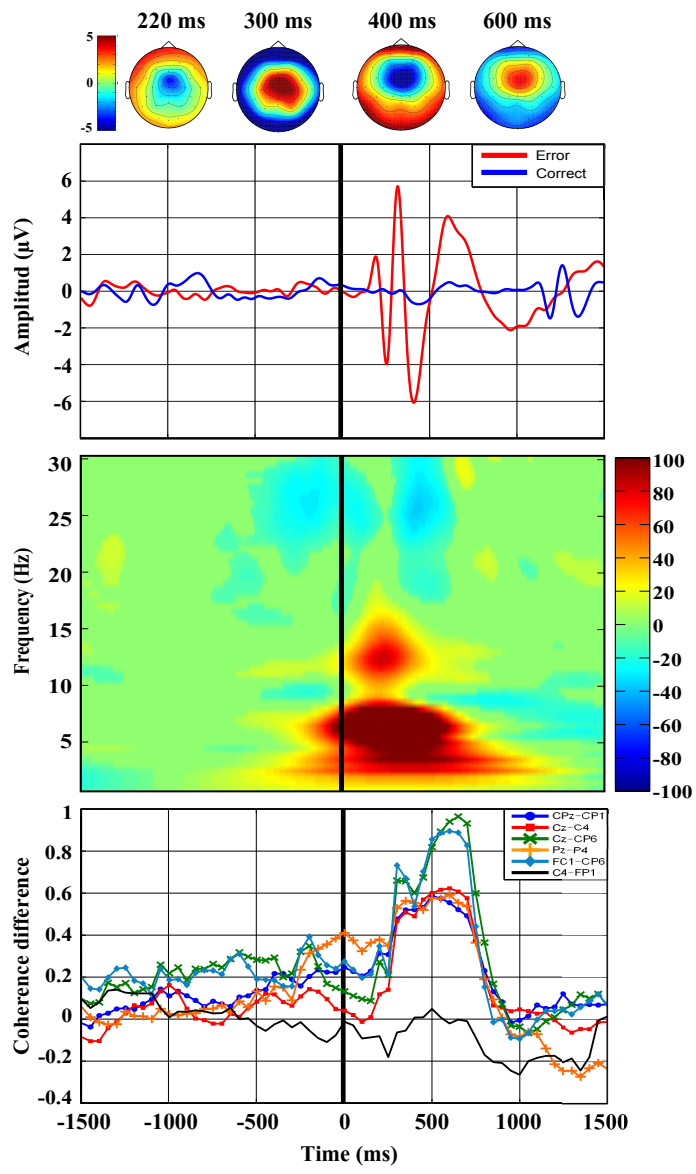

Fig. 4. From top to bottom: topographic representation of the most relevant peaks. Grand average of the signal at channel FCz. Time-frequency plots on channel $\mathrm{FCz}$ (red and blue represent significant ERS/ERD). Coherence evolution for sliding windows of $500 \mathrm{~ms}$ for the five-most representative electrode pairs and a random non-representative pair.
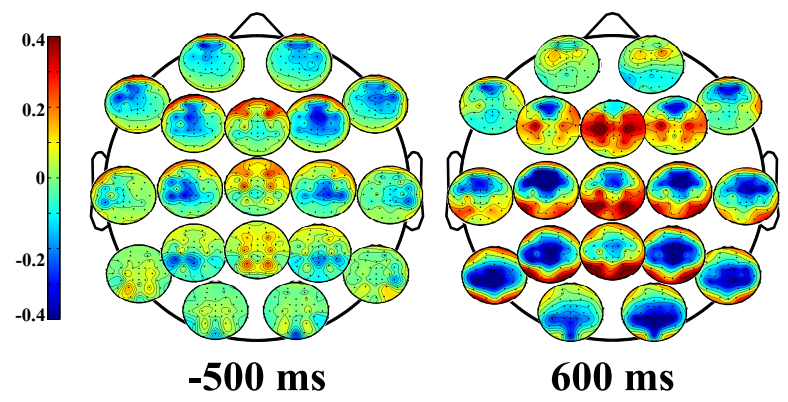

Fig. 5. Averaged Phase Slope Index for all pairs of channels on theta frequency band at -500 and $600 \mathrm{~ms}$ from the onset. The $i$ th small topographic plot is located at the $i$ th electrode pose, as indicated by the $i$ th row of the matrix $P S I_{i j}$. Red color represent outflow and blue color stands for inflow transference of information.

applications, or to study brain connectivity for erroneous actions where the onset of the event is not clearly presented.

\section{REFERENCES}

[1] M. Falkenstein, J. Hoormann, S. Christ, and J. Hohnsbein, "ERP components on reaction errors and their functional significance: A tutorial," Biological Psychology, vol. 51, pp. 87-107, 2000.
[2] C. B. Holroyd and M. G. H Coles, "The neural basis of human error processing: Reinforcement learning, dopamine, and the error-related negativity," 2002, pp. 679-709.

[3] P.W Ferrez and J.d.R. Millán, "Error-related EEG potentials generated during simulated Brain-Computer interaction," IEEE Trans Biomed Eng, vol. 55, no. 3, pp. 923-929, March 2008.

[4] J.d.R. Millán et al., "Combining brain-computer interfaces and assistive technologies: state-of-the-art and challenges," Front Neurosci, vol. 4, 2010.

[5] G. Schalk, J. R. Wolpaw, D. J. McFarland, and G. Pfurtscheller, "Eeg-based communication: presence of an error potential," Clinical Neurophysiology, vol. 111, no. 12, pp. 2138-2144, 2000.

[6] B. Blankertz, G. Dornhege, C. Schafer, R. Krepki, J. Kohlmorgen, K. R. Muller, V. Kunzmann, F. Losch, and G. Curio, "Boosting bit rates and error detection for the classification of fast-paced motor commands based on single-trial EEG analysis," IEEE Trans Neural Syst and Rehab Eng, vol. 11, no. 2, pp. 127-131, 2003.

[7] A. Llera, V. Gómez, and H.J. Kappen, "Adaptive classification on brain-computer interfaces using reinforcement signals," Neural Computation, vol. 24, no. 11, pp. 2900-2923, 2012.

[8] R. Chavarriaga and J.d.R. Millán, "Learning from EEG error-related potentials in noninvasive brain-computer interfaces," IEEE Trans Neural Syst Rehabil Eng, vol. 18, no. 4, pp. 381-388, 2010.

[9] I. Iturrate, L. Montesano, and J. Minguez, "Shared-control braincomputer interface for a two dimensional reaching task using eeg errorrelated potentials," in Int. Conf. of the IEEE Engineering in Medicine and Biology Society (EMBC). IEEE, 2013.

[10] T. Milekovic, T. Ball, A. Schulze-Bonhage, A. Aertsen, and C. Mehring, "Error-related electrocorticographic activity in humans during continuous movements," Journal of neural engineering, vol. 9, no. 2 , pp. $026007,2012$.

[11] Ren Xu, Ning Jiang, Chuang Lin, N. Mrachacz-Kersting, K. Dremstrup, and D. Farina, "Enhanced low-latency detection of motor intention from eeg for closed-loop brain-computer interface applications," Biomedical Engineering, IEEE Transactions on, vol. 61, no. 2, pp. 288-296, Feb 2014.

[12] M. X. Cohen, "Error-related medial frontal theta activity predicts cingulate-related structural connectivity.," NeuroImage, vol. 55, no. 3, pp. 1373-1383, 2011.

[13] J. Omedes, I. Iturrate, L. Montesano, and J. Minguez, "Using frequency-domain features for the generalization of eeg error-related potentials among different tasks," in Int. Conf. of the IEEE Engineering in Medicine and Biology Society (EMBC). IEEE, 2013.

[14] H. Zhang, R. Chavarriaga, M. K. Goel, L. Gheorghe, and J.d.R. Millan, "Improved recognition of error related potentials through the use of brain connectivity features," in Engineering in Medicine and Biology Society (EMBC), 2012 Annual International Conference of the IEEE. IEEE, 2012, pp. 6740-6743.

[15] S. F. Taylor, E. R. Stern, and W. J. Gehring, "Neural systems for error monitoring recent findings and theoretical perspectives," The Neuroscientist, vol. 13, no. 2, pp. 160-172, 2007.

[16] A. Schlögl, C. Keinrath, D. Zimmermann, R. Scherer, R. Leeb, and G. Pfurtscheller, "A fully automated correction method of EOG artifacts in EEG recordings," Clinical neurophysiology, vol. 118, no. 1, pp. 98-104, Jan. 2007.

[17] H. Akaike, "A new look at the statistical model identification," Automatic Control, IEEE Transactions on, vol. 19, no. 6, pp. 716723, Dec. 1974.

[18] G. Nolte, A. Ziehe, V. V. Nikulin, A. Schlögl, N. Krämer, T. Brismar, and K.R. Müller, "Robustly estimating the flow direction of information in complex physical systems," Phys. Rev. Lett., vol. 100, pp. 234101, Jun 2008.

[19] C. Andrew and G. Pfurtscheller, "Event-related coherence as a tool for studying dynamic interaction of brain regions," Electroencephalography and clinical Neurophysiology, vol. 98, no. 2, pp. 144-148, 1996.

[20] B. Graimann and G. Pfurtscheller, "Quantification and visualization of event-related changes in oscillatory brain activity in the timefrequency domain," Progress in brain research, vol. 159, pp. 79-97, 2006. 